\title{
Analysis to Determine Optimum Steam Pressure before Control Valves to Minimize Throttling Losses
}

\author{
Gaurav Masiwal $^{\# 1}$, P.S.Kumar ${ }^{* 2}$, Sumit Chaudhary ${ }^{* 3}$ \\ \# Operation and Efficiency Department, \\ Steag Operation and Maintenance Company Private Limited (SOMC), Andhra Pradesh, India \\ ${ }^{1}$ masiwalgaurav@gmail.com \\ * Power Plant Professional, \\ Steag Operation and Maintenance Company Private Limited (SOMC), Andhra Pradesh, India \\ 2 ps.kumar@somc.co.in \\ * Mechanical Department, Delhi Technological University, Delhi, India \\ ${ }^{3}$ mait.sumit@gmail.com
}

\begin{abstract}
In this paper, analysis has been done to determine how much optimum steam pressure should be maintained before control valves at part loads so that throttling losses would be minimum. Comparison has been made between existing values maintained at present and calculated values from proposed method. Results have shown that approximately $8 \mathrm{ksc}$ of pressure can be saved and process can be improved by adopting this method. This method can be proved useful to generate main steam pressure verses load curve. All data for analysis has been collected and evaluated from a 525MW operating unit of Bharat Heavy Electricals Limited.
\end{abstract}

Keyword - Sliding pressure mode; Throttling losses; Heat rate; optimum steam pressure; Control valves opening; Power Plant

\section{INTRODUCTION}

Electric energy market imposed a new way to operate coal power plant units. The tendency is to operate these units, not in base load (with constant and close to nominal load), but in semi base load (with important load variation). In constant pressure mode of operation, pressure of steam is maintained constant at turbine inlet by varying the control valve opening. In sliding pressure mode of operation, control valves opening remain constant, and the live steam pressures will vary as a function of the turbine load [1]. The main advantage of the sliding pressure mode over constant pressure mode are (1) Energy losses due to throttling are reduced, (2) Steam turbines efficiencies are improved at part loads as steam with higher enthalpy is available, in other words, superheated steam with higher available energy is available for conversion into work. With sliding pressure operating mode one can assure same flexibility and faster transition between loads comparable with constant pressure mode of operation [1].

Heat balance diagrams furnished by OEM at different loads with sliding pressure operation; provide values of main steam pressure before control valve with control valve wide open condition. Typical design heat balance diagrams values of main steam pressure before control valves at 100\% load, $80 \%$ load, $65 \%$ load, and $50 \%$ load in constant pressure mode and sliding pressure mode is shown in Table I. But in actual scenario, machine cannot be operated in valve wide open condition as some margins are to be made available in valve opening to facilitate grid demand during varying frequency conditions. Also there may be some situations like coal feeder tripping; coal calorific value variation. During such situations, major deviations between actual load and declared capacity cannot be afforded considering the commercial impact of such deviation. In case of valve wide open conditions, if coal feeder trips or coal higher heating value varies, it would be difficult to restore the system without taking oil support, as main steam pressure either drops drastically due to feeder tripping or shoots up because of good higher heating value of coal, leading to unstable conditions like pressure release through safety valves.

Mainly three types of operating modes are adopted in power plants: (1) The Turbine follow mode (Constant Pressure Control Mode), (2) Boiler Follow mode (Variable Pressure Control Mode), (3) Coordinated Machine control (CMC mode).

(1) The Turbine follow mode (Constant Pressure Control Mode) - In turbine follow mode the turbine output is varied so as to maintain a constant set pressure at inlet of turbine control valves. Load set point is given to Boiler. MW error between set point and actual MW is provided a correction to Boiler demand. Thus the turbine governing valve open or close to regulate turbine inlet pressure.

(2) Boiler Follow mode (Variable Pressure Control Mode): In boiler follow mode, the load set point is given to the turbine and the boiler demand is varied in accordance with the load set point given to turbine. Turbine 
inlet pressure varies depending on the actual energy output from Boiler. The error between set pressure at turbine inlet and actual pressure is used as a corrective signal for boiler demand.

(3) CMC Mode (Coordinated machine control mode): It is the combination of both of turbine follow mode and boiler follow mode to achieve optimum output of plant using maximum efficiency of all equipment in the entire operating range. In CMC mode as both pressure and load are maintained it depends on the experience of the operator as to what pressure is to be set before control valves to obtain maximum efficiency. The OEM also provide a load versus pressure curve which is in built in the control loops. While developing this curve, priority is given by OEM to set the pressure so as to obtain better response of the control parameters (minimum deviation between set and actual value) during varying load conditions. If this sliding pressure value is more than what should be maintained (including margins for exigency), then energy is wasted unnecessarily in throttling without actually knowing about it.

George Darie, Horia Peteu, Gabriel Negreanu, and Viorel Gherghina [1] have shown the advantages of sliding pressure mode of operation over the constant pressure mode of operation. Gerald Weber Commonwealth Edison Company [3] has done analysis to determine the steam flow rate for a particular load at constant valve opening. Yong Hu, Ji-zhen Liu, De-Liang Zeng, Wei Wang, and Ya-zhe Li [4] have shown the calculation of governing stage. Gerard Kosman, Henryk Lukowicz, krzysztof Nawrat, and Wojciech Kosman [5] have shown the benefits of full sliding pressure and partial sliding pressure for a specific load. S.C.Kaushik, V.Siva Reddy, S.K.Tyagi [6] has done energy and exergy analysis and shown the main area of energy/exergy losses in thermal power plant. Sairam Adibhatla, S.C.Kaushik [7] has compared energy and exergy losses for constant pressure mode and sliding pressure mode for a supercritical unit. Ankur Geete, A.I. Khandwawala [8] has shown the effects of different steam temperature on cycle efficiency by keeping steam pressure constant. Marc A. Rosen [9] have explained the potential thermodynamic losses by exergy approach. Janulis, V.J [10] have shown the benefits of sliding pressure in turbine heat rate. Silvestri, G.J. JR-Aanstad, O.J.Ballantyne, J.J. [11] have analysed the sliding pressure operation for thermal power units. Robert A.Leffler, Craig R.Bradshaw, Eckhard A.Groll, Suresh V.Garimella [12] have described the heat rejection methods. Wei Wang, Lu Li, Dongteng Long, Tizhen Liu, Deliang Zenf, Can Cui [13] have analysed the response of coordinated control system for 1000MW thermal plant. Wen Tan, Fang Fang, Liang Tian, Caifen Fu, Jizhen Liu [14] have shown and analysed the linear control behaviour for both boiler-turbine. Omendra Kumar Singh, S.C.Kaushik [15] have shown the influence of various factors which influence exergy performance of a steam power plant.

The objective of this paper is to analyze the throttling losses and to suggest the optimum or near to possible optimum value of main steam pressure which should be maintained before control valves in the entire operating range, so that deviations are less in any exigency like coal calorific value variations or load variations due to frequency variations and in addition reduce throttling losses comparatively. Difference between indicated DCS sliding pressure, main steam pressure maintained by operators, calculated/optimum main steam pressure and load dependent first stage pressure (at rated temperatures) are observed. For calculating optimized main steam pressure before control valves thermodynamic heat balance approach across control valves has been used.

\section{PLANT DESCRIPTION}

The power plant has a total installed power capacity of $1050 \mathrm{MW}$. The power house consist of two steam turbines units of $525 \mathrm{MW}$ each. The schematic diagram of one $525 \mathrm{MW}$ unit is shown in Fig.1. This unit employs reheating and regenerative feed water heating system. Feed water heating is carried out in two stages of high pressure heaters (HPH-6, HPH-5) and three stages of low pressure heaters (LPH-3, LPH-2, LPH-1) along with one deaerating heat exchanger. Steam is superheated to $170 \mathrm{bar}$ and $537^{\circ} \mathrm{C}$ in the steam generator and fed to the high pressure turbine. The high pressure turbine exhaust then goes to reheater where steam is reheated to $537^{\circ} \mathrm{C}$ and enters to intermediate pressure turbine. Low pressure turbine exhaust is sent to condenser. Condensate collected in hotwell is extracted and pumped by condensate extraction pumps (CEP) to the low pressure heaters. Condensate water after LPH-3 goes to deaerator and then pumped by boiler feed pumps to the high pressure heaters. And thus whole cycle repeats again.

\section{A. Nomenclature}

\begin{tabular}{|ll|}
\hline $\mathrm{m}_{1}, \mathrm{~m}_{2}$ & Steam flow rate at entry and exit of control valves $(\mathrm{kg} / \mathrm{sec})$ \\
$\mathrm{u}_{1}, \mathrm{u}_{2}$ & Specific internal energy of steam at entry and exit of control valves $(\mathrm{kJ} / \mathrm{kg})$ \\
$\mathrm{h}_{1}, \mathrm{~h}_{2}$ & Specific enthalpy of steam at entry and exit of control valves $(\mathrm{kJ} / \mathrm{kg})$ \\
$\mathrm{V}_{1}, \mathrm{~V}_{2}$ & Velocity of steam at entry and exit of control valves $(\mathrm{m} / \mathrm{sec})$ \\
$\mathrm{z}_{1}, \mathrm{z}_{2}$ & Elevations at entry and exit of system respectively $(\mathrm{m})$. \\
$\mathrm{v}_{1}, \mathrm{v}_{2}$ & Specific volumes at entry and exit of control valves respectively $\left(\mathrm{m}^{3} / \mathrm{kg}\right)$. \\
\hline
\end{tabular}




\begin{tabular}{|ll|}
\hline $\mathrm{s}_{1}, \mathrm{~s}_{2}$ & Specific entropy at entry and exit of control valves respectively $(\mathrm{kJ} / \mathrm{kg}-\mathrm{K})$. \\
$\mathrm{S}_{\mathrm{gen}}$ & Entropy generation $(\mathrm{kJ} / \mathrm{kg}-\mathrm{k})$ \\
$\delta$ & Specific heat ratio \\
$\mathrm{A}_{1}, \mathrm{~A}_{2}$ & Area of pipe before control valve and control valve opening area respectively $\left(\mathrm{m}^{2}\right)$ \\
$\Delta \mathrm{Q}$ & Heat interactions $(\mathrm{kJ} / \mathrm{kg})$ \\
$\Delta \mathrm{W}$ & Work interactions $(\mathrm{kJ} / \mathrm{kg})$ \\
$\mathrm{MS}$ & Main steam \\
$\mathrm{SH}$ & Superheater \\
$\mathrm{DCS}$ & Distributed Control system \\
OEM & Original Equipment Manufacturer \\
RGMO & Restricted Governor Mode Operation \\
\hline
\end{tabular}

Table 1. Design Values of Main Steam in Constant Pressure and Sliding Pressure Mode of Operations ( $0 \%$ Make Up)

\begin{tabular}{|c|l|c|c|c|c|c|c|c|c|}
\hline $\begin{array}{c}\text { S. } \\
\text { No }\end{array}$ & Parameters & \multicolumn{4}{|c|}{ Constant pressure mode of } & \multicolumn{4}{c|}{ Sliding pressure mode of } \\
operation \\
\hline 1 & Power output & $\begin{array}{r}525 \\
\text { MW }\end{array}$ & $\begin{array}{c}420 \\
\text { MW }\end{array}$ & $\begin{array}{c}315 \\
\text { MW }\end{array}$ & $\begin{array}{c}262.5 \\
\text { MW }\end{array}$ & $\begin{array}{c}525 \\
\text { MW }\end{array}$ & $\begin{array}{c}420 \\
\text { MW }\end{array}$ & $\begin{array}{c}315 \\
\text { MW }\end{array}$ & $\begin{array}{c}262.5 \\
\text { MW }\end{array}$ \\
\hline 2 & Main steam flow $(\mathrm{kg} / \mathrm{s})$ & 436 & 348 & 264 & 223 & 434 & 343 & 257 & 216 \\
\hline 3 & Main steam pressure $(\mathrm{bar})$ & 170 & 170 & 170 & 170 & 160 & 128 & 96.99 & 81.94 \\
\hline 4 & Main steam temperature $\left({ }^{\circ} \mathrm{C}\right)$ & 537 & 537 & 537 & 537 & 537 & 537 & 537 & 537 \\
\hline 5 & Reheater flow $(\mathrm{kg} / \mathrm{s})$ & 390 & 315 & 241 & 205 & 389 & 310 & 234 & 198 \\
\hline 6 & Reheated steam pressure $(\mathrm{bar})$ & 40.43 & 32.7 & 25.09 & 21.37 & 40.29 & 32.2 & 24.46 & 20.7 \\
\hline 7 & Reheated steam temperature $\left({ }^{\circ} \mathrm{C}\right)$ & 537 & 537 & 537 & 537 & 537 & 537 & 537 & 537 \\
\hline 8 & Feed water flow $(\mathrm{kg} / \mathrm{s})$ & 436 & 348 & 264 & 223 & 434 & 343 & 257 & 216 \\
\hline 9 & $\begin{array}{l}\text { Feed water inlet temperature to } \\
\text { boiler }\left({ }^{\circ} \mathrm{C}\right)\end{array}$ & 253.4 & 242.5 & 229.3 & 221.6 & 253.3 & 242.4 & 229 & 221.2 \\
\hline
\end{tabular}

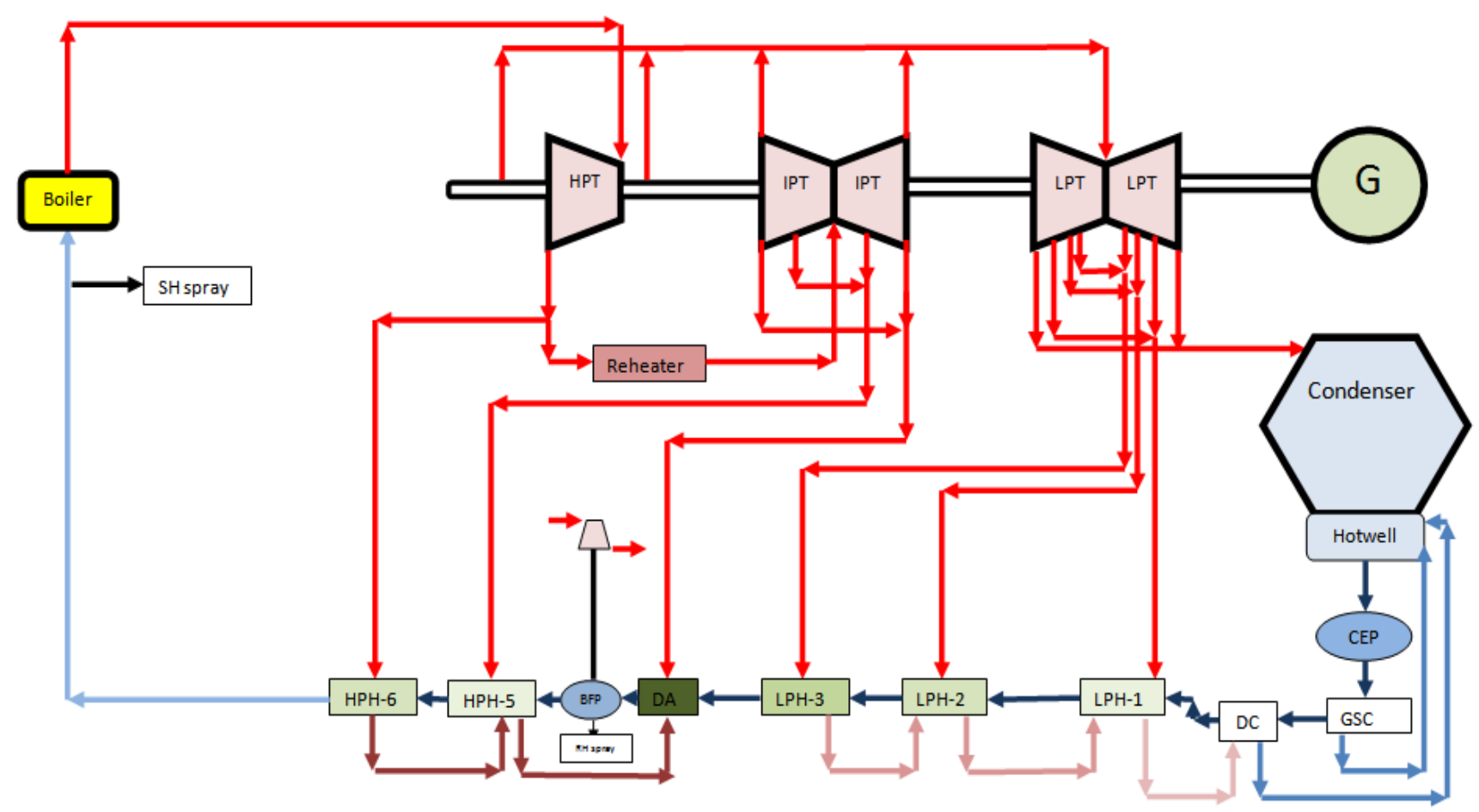

Fig.1. Schematic diagram of power plant. 


\section{III.CALCULATION METHODOLOGY}

Throttling process is highly irreversible process, and amount of throttling depends upon the difference in pressure before control valves and downstream pressure. Downstream pressure will be fixed for a particular load, if the steam temperatures are constant. So more the pressure difference across the throttle valve more will be the throttling loss. Mathematically we can express the relation as given below [3].

Assumptions made: Steady flow, Adiabatic conditions (No heat transfer), Velocity in x-direction (for making simple conclusion). Using T-ds equations across control a valve as shown in Fig 2.

$\mathrm{Tds}=\mathrm{dh}-\mathrm{vdp}$

For particular direction, we can differentiate with $\mathrm{x}$-direction;

$\mathrm{T} \underline{\mathrm{ds}}=\underline{\mathrm{dh}}-\underline{\mathrm{vdp}}$

$\mathrm{dx} \quad \mathrm{dx} \quad \mathrm{dx}$

$\mathrm{ds} / \mathrm{dx}$ or $\mathrm{S}_{\mathrm{gen}}$ is the entropy generation/change of entropy with respect to change in distance, and it is always positive as our entropy is increasing in $\mathrm{X}$-direction.

As throttling process is isenthalpic process $(\mathrm{dh}=0)$, equation can be rearranged as

$\mathrm{T}^{*} \mathrm{~S}_{\mathrm{gen}}=-\underline{\mathrm{vdp}} \quad\left(\mathrm{dp}=\mathrm{p}_{2}-\mathrm{p}_{1}\right.$ as $\mathrm{p}_{2}$ would be less than $\mathrm{p}_{1}$, dp will always negative $)$

$\mathrm{dx}$

$\mathrm{S}_{\mathrm{gen}}=-\underline{\mathrm{v}^{*} \mathrm{dp}} \quad$ (entropy generation is always positive)

$\mathrm{T} \mathrm{dx}$

This relation implies that across a control valve, if pressure drop is more, then associated entropy generation would be more. Means degradation of quality of energy will be more. For minimizing this entropy generation our pressure drop across control valve should be less. Steam parameters considered for calculation is given in Table II.

According to first law of thermodynamics:

$$
\mathrm{m}_{1} *\left(\mathrm{~h}_{1}+\mathrm{V}_{1}^{2} / 2+\mathrm{gZ} \mathrm{Z}_{1}\right)+\Delta \mathrm{Q}=\mathrm{m}_{2} *\left(\mathrm{~h}_{2}+\mathrm{V}_{2}^{2} / 2+\mathrm{gZ} \mathrm{Z}_{2}\right)+\Delta \mathrm{W}
$$

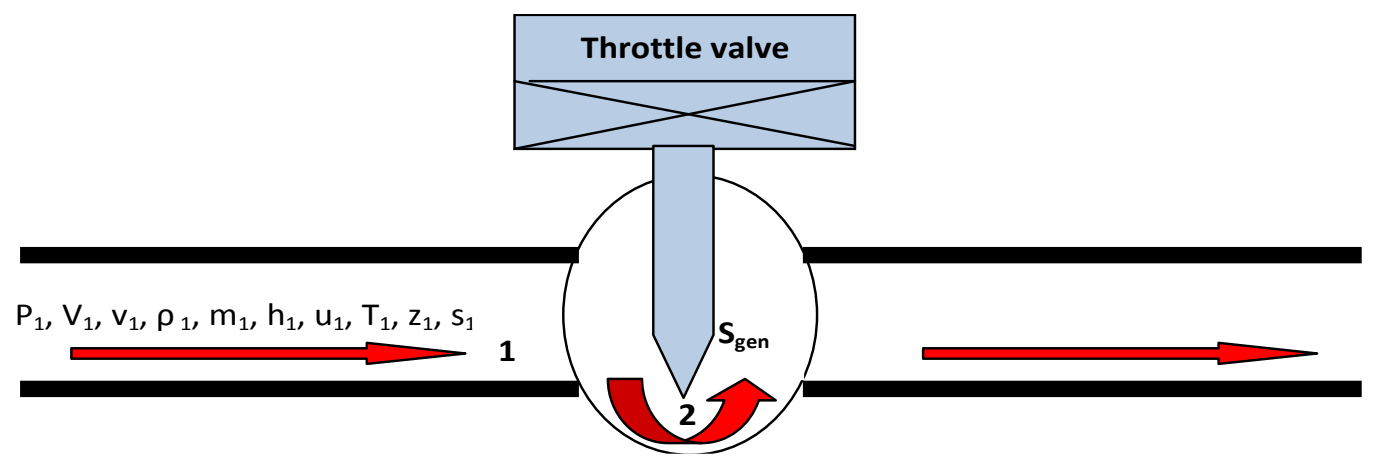

$P_{2}, V_{2}, v_{2}, \rho_{2}, m_{2}, h_{2}, u_{2}, T_{2}, z_{2}, s_{2}$

Fig.2. Heat and mass balance across control valve

Assumptions:

1. As there is no work or heat interactions during throttling process $(\Delta \mathrm{Q}=0$ and $\Delta \mathrm{W}=0)$.

$\mathrm{m}_{1} *\left(\mathrm{~h}_{1}+\mathrm{V}_{1}^{2} / 2+\mathrm{gZ1}\right)=\mathrm{m}_{2} *\left(\mathrm{~h}_{2}+\mathrm{V}_{2}^{2} / 2+\mathrm{gZ} 2\right)$

2. On applying continuity equation $\mathrm{m}_{1}=\mathrm{m}_{2}$,

$\left(\mathrm{h}_{1}+\mathrm{V}_{1}^{2} / 2+\mathrm{gZ}\right)=\left(\mathrm{h}_{2}+\mathrm{V}_{2}^{2} / 2+\mathrm{gZ}_{2}\right)$

3. As it is on horizontal position $Z_{1}=Z_{2}$

$\mathrm{h}_{1}+\mathrm{V}_{1}^{2} / 2=\mathrm{h}^{2}+\mathrm{V}_{2}^{2} / 2$

4. As state point 2 is at vena-contracta, then we can write $V_{2} \gg>>V_{1}$ which implies $V_{2}{ }^{2}>>>>>>V^{2}$, so we can just neglect $\mathrm{V}^{2}$.

$\mathrm{h}_{1}=\mathrm{h}_{2}+\mathrm{V}_{2}^{2} / 2$

As $h=u+p v$

$\mathrm{u}_{1}+\mathrm{p}_{1} \mathrm{v}_{1}=\mathrm{u}_{2}+\mathrm{p}_{2} \mathrm{v}_{2}+\mathrm{V}_{2}{ }^{2} / 2$ 
Table II. Steam Parameters at Various Loading Conditions

\begin{tabular}{|c|c|c|c|c|c|c|c|c|c|c|c|}
\hline \multicolumn{12}{|c|}{ Load (MW) } \\
\hline S.No & Parameters & 338 & 357 & 367 & 378 & 394 & 404 & 428 & 441 & 477 & 500 \\
\hline 1 & $\begin{array}{l}\text { MS press at } \mathrm{SH} \mathrm{O} / \mathrm{L} \\
-\quad \text { left (bar) }\end{array}$ & 133.6 & 135.7 & 136.3 & 131.7 & 137.1 & 137.9 & 144.0 & 147.2 & 155.7 & 160.3 \\
\hline 2 & $\begin{array}{l}\text { MS press at } \mathrm{SH} \mathrm{O} / \mathrm{L} \\
\text { - right (bar) }\end{array}$ & 134 & 136 & 136 & 132 & 137 & 138 & 144 & 147 & 156 & 160 \\
\hline 3 & $\begin{array}{l}\text { MS temp at SH O/L - } \\
\text { left }\left({ }^{\circ} \mathrm{C}\right)\end{array}$ & 539 & 542 & 542 & 541 & 540 & 539 & 540 & 537 & 541 & 538 \\
\hline 4 & $\begin{array}{l}\mathrm{MS} \text { temp at } \mathrm{SH} \mathrm{O} / \mathrm{L} \\
\text { - right }\left({ }^{\circ} \mathrm{C}\right)\end{array}$ & 539.9 & 541.8 & 541.8 & 540.8 & 540.1 & 539.6 & 540.8 & 538.9 & 541.0 & 537.4 \\
\hline 5 & MS Flow (kg/s) & 275.4 & 290.1 & 298.1 & 304.3 & 319.2 & 325.7 & 344.7 & 360.5 & 388.4 & 406.0 \\
\hline 6 & $\begin{array}{l}\text { DCS indicated } \\
\text { sliding pressure (bar) }\end{array}$ & 117.5 & 122.7 & 125.7 & 128.7 & 133.1 & 135.8 & 141.9 & 145.6 & 156.1 & 160.6 \\
\hline 7 & $\begin{array}{l}\text { MS press at MS } \\
\text { header (bar) }\end{array}$ & 131.4 & 133.3 & 133.8 & 129.0 & 134.3 & 135.1 & 141.0 & 144.1 & 152.3 & 156.8 \\
\hline 8 & $\begin{array}{l}\text { MS temp at MS } \\
\text { header }\left({ }^{\circ} \mathrm{C}\right)\end{array}$ & 539 & 541 & 541 & 540 & 539 & 538 & 539 & 536 & 539 & 536 \\
\hline 9 & $\begin{array}{l}\text { Throttle control valve } \\
\text { opening - left }(\%)\end{array}$ & 66.1 & 67.9 & 76.1 & 71.2 & 72.4 & 73.8 & 77.4 & 79.6 & 71.7 & 90.1 \\
\hline 10 & $\begin{array}{l}\text { Throttle control valve } \\
\text { opening - right }(\%)\end{array}$ & 66.6 & 68.4 & 76.7 & 71.7 & 72.9 & 74.3 & 78.0 & 80.3 & 72.2 & 90.8 \\
\hline 11 & First stage press (bar) & 102.6 & 107.9 & 110.9 & 112.6 & 117.6 & 121.1 & 128.0 & 133.3 & 143.4 & 150.7 \\
\hline
\end{tabular}

For an ideal gas,

We can write $\mathrm{u}=\mathrm{C}_{\mathrm{v}} * \mathrm{~T}$

$\mathrm{C}_{\mathrm{v}} * \mathrm{~T}_{1}+\mathrm{p}_{1} \mathrm{v}_{1}=\mathrm{C}_{\mathrm{v}} * \mathrm{~T}_{2}+\mathrm{p}_{2} \mathrm{v}_{2}+\mathrm{V}_{2}^{2} / 2$

$\mathrm{V}_{2}^{2} / 2=\mathrm{C}_{\mathrm{v}} * \mathrm{~T}_{1}+\mathrm{p}_{1} \mathrm{v}_{1}-\mathrm{C}_{\mathrm{v}} * \mathrm{~T}_{2}-\mathrm{p}_{2} \mathrm{v}_{2}$

$\mathrm{V}_{2}^{2} / 2=\mathrm{C}_{\mathrm{v}} *\left(\mathrm{~T}_{1}-\mathrm{T}_{2}\right)+\mathrm{p}_{1} \mathrm{v}_{1}-\mathrm{p}_{2} \mathrm{v}_{2}$

As per ideal gas equation; $\mathrm{pv}=\mathrm{RT}$ and we can write it as $\mathrm{pv} / \mathrm{R}=\mathrm{T}$

So, $\mathrm{V}_{2}^{2} / 2=\mathrm{C}_{\mathrm{v}} / \mathrm{R} *\left(\mathrm{p}_{1} \mathrm{v}_{1}-\mathrm{p}_{2} \mathrm{v}_{2}\right)+\mathrm{p}_{1} \mathrm{v}_{1}-\mathrm{p}_{2} \mathrm{~V}_{2}$

We can write $\mathrm{C}_{\mathrm{p}}-\mathrm{C}_{\mathrm{v}}=\mathrm{R}$

$\mathrm{C}_{\mathrm{p}} / \mathrm{C}_{\mathrm{v}}-1=\mathrm{R} / \mathrm{C}_{\mathrm{v}}$

$\delta-1=\mathrm{R} / \mathrm{C}_{\mathrm{v}}$

$\mathrm{V}_{2}^{2} / 2=\left(\mathrm{p}_{1} \mathrm{~V}_{1}-\mathrm{p}_{2} \mathrm{~V}_{2}\right) /(\delta-1)+\mathrm{p}_{1} \mathrm{~V}_{1}-\mathrm{p}_{2} \mathrm{~V}_{2}$

$\mathrm{V}_{2}^{2} / 2=\left(\mathrm{p}_{1} \mathrm{v}_{1}-\mathrm{p}_{2} \mathrm{~V}_{2}\right) * \delta /(\delta-1)$

As it is an adiabatic process; $\mathrm{pv}^{\delta}=$ constant

$\mathrm{p}_{1}{ }^{*} \mathrm{v}_{1}{ }^{\delta}=\mathrm{p}_{2} * \mathrm{v}_{2}{ }^{\delta}$

$\left(\mathrm{v}_{2} / \mathrm{v}_{1}\right)=\left(\mathrm{p}_{1} / \mathrm{p}_{2}\right)^{1 / \delta}$

$\mathrm{v}_{2}=\mathrm{v}_{1} *\left(\mathrm{p}_{1} / \mathrm{p}_{2}\right)^{1 / \delta}$

$\mathrm{p}_{1} *\left(\mathrm{RT}_{1} / \mathrm{p}_{1}\right)^{\delta}=\mathrm{p}_{2} *\left(\mathrm{RT}_{2} / \mathrm{p}_{2}\right)^{\delta}$

$\mathrm{p}_{1}{ }^{1-\delta} * \mathrm{~T}_{1}{ }^{\delta}=\mathrm{p}_{2}{ }^{1-\delta} * \mathrm{~T} 2^{\delta}$

$\left(\mathrm{T}_{2} / \mathrm{T}_{1}\right)=\left(\mathrm{p}_{2} / \mathrm{p}_{1}\right)^{(\delta-1) / \delta}$ 
Now from Avogadro's law: $\mathrm{p}_{1} \mathrm{v}_{1} / \mathrm{T}_{1}=\mathrm{p}_{2} \mathrm{v}_{2} / \mathrm{T}_{2}$

$\mathrm{p}_{2} \mathrm{v}_{2}=\mathrm{p}_{1} \mathrm{v}_{1} * \mathrm{~T}_{2} / \mathrm{T}_{1}$

$\mathrm{p}_{2} \mathrm{~V}_{2}=\mathrm{p}_{1} \mathrm{v}_{1} *\left(\mathrm{p}_{2} / \mathrm{p}_{1}\right)^{(\delta-1) / \delta}$

$\mathrm{V}_{2}^{2} / 2=\delta /(\delta-1) *\left[\mathrm{p}_{1} \mathrm{v}_{1}-\mathrm{p}_{1} \mathrm{v}_{1} *\left(\mathrm{p}_{2} / \mathrm{p}_{1}\right)^{(\delta-1) / \delta)}\right]$

$\mathrm{V}_{2}^{2} / 2=\delta /(\delta-1) * \mathrm{p}_{1} \mathrm{~V}_{1} *\left[1-\left(\mathrm{p}_{2} / \mathrm{p}_{1}\right)^{(\delta-1) / \delta)}\right]$

Now; $m=\rho^{*} A^{*} V$

$\mathrm{m}=(\mathrm{A} * \mathrm{~V}) / \mathrm{v}$

Let $\mathrm{z}=\mathrm{p}_{2} / \mathrm{p}_{1}$

$\left(\mathrm{m}_{2}{ }^{2} * \mathrm{v}_{2}{ }^{2}\right) /\left(2 * \mathrm{~A}_{2}^{2}\right)=\delta /(\delta-1)^{*} \mathrm{p}_{1} \mathrm{v}_{1} *\left[1-(\mathrm{z})^{(\delta-1) / \delta}\right]$

$\left(\mathrm{m}_{2}{ }^{2 *} \mathrm{v}_{2}{ }^{2}\right)=2 * \mathrm{~A}_{2}{ }^{2} * \delta /(\delta-1)^{*} \mathrm{p}_{1} \mathrm{v}_{1} *\left[1-(\mathrm{z})^{(\delta-1) / \delta}\right]$

$\mathrm{m}_{2}{ }^{2}=2 * \mathrm{~A}_{2}{ }^{2} / \mathrm{v}_{2}{ }^{2} * \delta /(\delta-1) * \mathrm{p}_{1} \mathrm{v}_{1} *\left[1-(\mathrm{z})^{(\delta-1) / \delta}\right]$

$\mathrm{m}_{2}^{2}=\frac{2 * \mathrm{~A}_{2}^{2}}{2} \frac{2 /(\delta-1) * \mathrm{p}_{1}}{\mathrm{v}_{1}{ }_{1} *\left(\mathrm{p}_{1} / \mathrm{p}_{2}\right)^{2 / \delta}} \frac{\left.1-(\mathrm{z})^{(\delta-1) / \delta)}\right]}{\mathrm{v}_{1}}$

$\mathrm{m}_{2}^{2}=\frac{2 * \mathrm{~A}_{2}}{2} \frac{{ }^{2} * \delta /(\delta-1) * \mathrm{p}_{1} *\left[1-(\mathrm{z})^{(\delta-1) / \delta}\right]}{\mathrm{v}_{1} *\left(\mathrm{p}_{1} / \mathrm{p}_{2}\right)^{2 / \delta}}$

$\mathrm{m}_{2}{ }^{2}=2 * \mathrm{~A}_{2}{ }^{2} * \delta /(\delta-1) *\left(\mathrm{p}_{1} / \mathrm{v}_{1}\right)^{*} \mathrm{z}^{2 / \delta} *\left[1-(\mathrm{z})^{(\delta-1) / \delta}\right]$

$\mathrm{m}_{2}{ }^{2}=2 * \mathrm{~A}_{2}^{2} * \delta /(\delta-1) *\left(\mathrm{p}_{1} / \mathrm{v}_{1}\right) *\left[(\mathrm{z})^{2 / \delta}-(\mathrm{z})^{(\delta+1) / \delta}\right]$

$\mathrm{m}_{2}=\operatorname{sqrt}\left\{2 * \mathrm{~A}_{2}{ }^{2} * \delta /(\delta-1) *\left(\mathrm{p}_{1} / \mathrm{v}_{1}\right) *\left[(\mathrm{z})^{2 / \delta}-(\mathrm{z})^{(\delta+1) / \delta}\right]\right\}$

For a particular load, if valve position stays constant then $\mathrm{p}_{2} / \mathrm{p}_{1}$ (i.e. $\mathrm{z}$ ), $\mathrm{A}_{2}$ and $\delta$ remains constant (let us say $\mathrm{K})$. Therefore for constant valve position flow rate is given by

$\mathrm{m}_{2}=\mathrm{K} * \operatorname{sqrt}\left(\mathrm{p}_{1} / \mathrm{v}_{1}\right)$

$\mathrm{K}=\operatorname{sqrt}\left\{2 * \mathrm{~A}_{2}^{2} * \delta /(\delta-1)^{*}\left[(\mathrm{z})^{2 / \delta}-(\mathrm{z})^{(\delta+1) / \delta)}\right]\right\}$

SI unit of ' $\mathrm{K}$ ' value is $\mathrm{m}^{2}$ and signifies the valve opening area.

\section{IV.RESULT AND DISCUSSIONS}

Difference between actual main steam pressure maintained before control valves, OEM set sliding pressure, first stage pressure and optimized main steam pressure which should be maintained in order to minimize throttling losses are shown in Fig 3. Optimized main steam pressure as shown in Table III has considered 8 ksc as a backup pressure over first stage pressure which will ensure minimum disturbance during RGMO mode and will take care of correction of $\pm 25 \mathrm{MW}$ according to grid demand. At $338 \mathrm{MW}$ load, OEM set sliding pressure is showing 117.5 bar, but actual main steam header pressure maintained by operators is 131.4 bar, optimized main steam pressure by calculations comes out to 111.4 bar and first stage pressure at $338 \mathrm{MW}$ load is 102.6 bar. First stage pressure measured is after first stage (most preferred tapping in turbines), hence pressure before first stage would be more than $102.6 \mathrm{ksc}$, further in throttling there would be some pressure loss, so in calculation we have taken $8 \mathrm{ksc}$ of pressure as a backup which will include all losses from before control valves to first stage and exigencies too. Still calculated main steam pressure which should be maintained before control valves is lesser than what is indicated by sliding pressure curve (set by OEM) and the actual pressure maintained in header by the operator. Difference between optimized and maintained main steam pressure would increase throttling losses. By adopting this method analysis, throttling losses can be minimized at part load and a most probable optimized main steam pressure can be determined which should be maintained before control valves in order to ensure minimum throttling in system.

\section{Conclusions}

By the above methodology, we can judge whether the main steam pressure maintained before control valve is optimum or not. If higher pressure is maintained before control valves then unnecessarily energy will be wasted in throttling. This method can be used to generate pressure verses load curve with real time parameters. By optimizing main steam pressure before control valves one can improve turbine cycle heat rate and unit cycle heat rate. As boiler duty will be less for the same power generation and less coal will be burnt. In this case control valve is not fixed but optimization of control valves opening is done. For ' $\mathrm{K}$ ' constant of control valves, extrapolation curve has been used in which value of ' $K$ ' constant will be a function of main steam flow. ' $K$ ' constant values are derived for given HBD's conditions by taking pressure before control valves 8 ksc more than that first stage pressure. Then a curve of ' $\mathrm{K}$ ' constant values and main steam flow has been drawn so that a generalized equation can be made and just by putting MS flow we can get ' $\mathrm{K}$ ' constant value for any loading 
condition. Optimum main steam pressure obtained by calculation should be treated as target main steam pressure and efforts should be made so that throttling losses should be minimum. By optimizing main steam pressure extra benefits can be obtained both from turbine and boiler when operating unit at part load. Further by lowering boiler drum pressure, pipe losses or steam side friction losses will reduce which will also increase overall cycle efficiency.

Table III. Optimized Main Steam Pressure at Different Loading Conditions

\begin{tabular}{|c|l|c|c|c|c|c|c|c|c|c|c|}
\hline & \multicolumn{10}{|c|}{ Load (MW) } \\
\hline S.No & \multicolumn{1}{|c|}{ Parameters } & 338 & 357 & 367 & 378 & 394 & 404 & 428 & 441 & 477 & 500 \\
\hline 1 & MS Flow (kg/s) & 275.4 & 290.1 & 298.1 & 304.3 & 319.2 & 325.7 & 344.7 & 360.5 & 388.4 & 406.0 \\
\hline 2 & First stage press (bar) & 102.6 & 107.9 & 110.9 & 112.6 & 117.6 & 121.1 & 128.0 & 133.3 & 143.4 & 150.7 \\
\hline 3 & 'K' constant (m) & 4.5569 & 4.5732 & 4.5821 & 4.5891 & 4.6060 & 4.6137 & 4.6373 & 4.6585 & 4.7014 & 4.7328 \\
\hline 4 & $\begin{array}{l}\text { Calculated MS press } \\
\text { before control valves } \\
\text { including margins } \\
\text { (bar) }\end{array}$ & 111.4 & 116.6 & 119.5 & 121.7 & 126.8 & 129.1 & 135.6 & 140.8 & 149.9 & 155.3 \\
\hline 5 & $\begin{array}{l}\text { Difference between } \\
\text { calculated and first } \\
\text { stage pressure (bar) }\end{array}$ & 8.8 & 8.7 & 8.6 & 9.0 & 9.2 & 7.9 & 7.5 & 7.6 & 6.4 & 4.6 \\
\hline 6 & $\begin{array}{l}\text { Difference between } \\
\text { calculated and DCS } \\
\text { indicated sliding } \\
\text { pressure (bar) }\end{array}$ & 6.07 & 6.06 & 6.24 & 7.09 & 6.31 & 6.71 & 6.37 & 4.80 & 6.26 & 5.24 \\
\hline 7 & $\begin{array}{l}\text { Difference between } \\
\text { calculated and } \\
\text { maintained pressure } \\
\text { (bar) }\end{array}$ & 20.0 & 16.7 & 14.4 & 7.4 & 7.5 & 6.0 & 5.5 & 3.2 & 2.5 & 1.4 \\
\hline
\end{tabular}

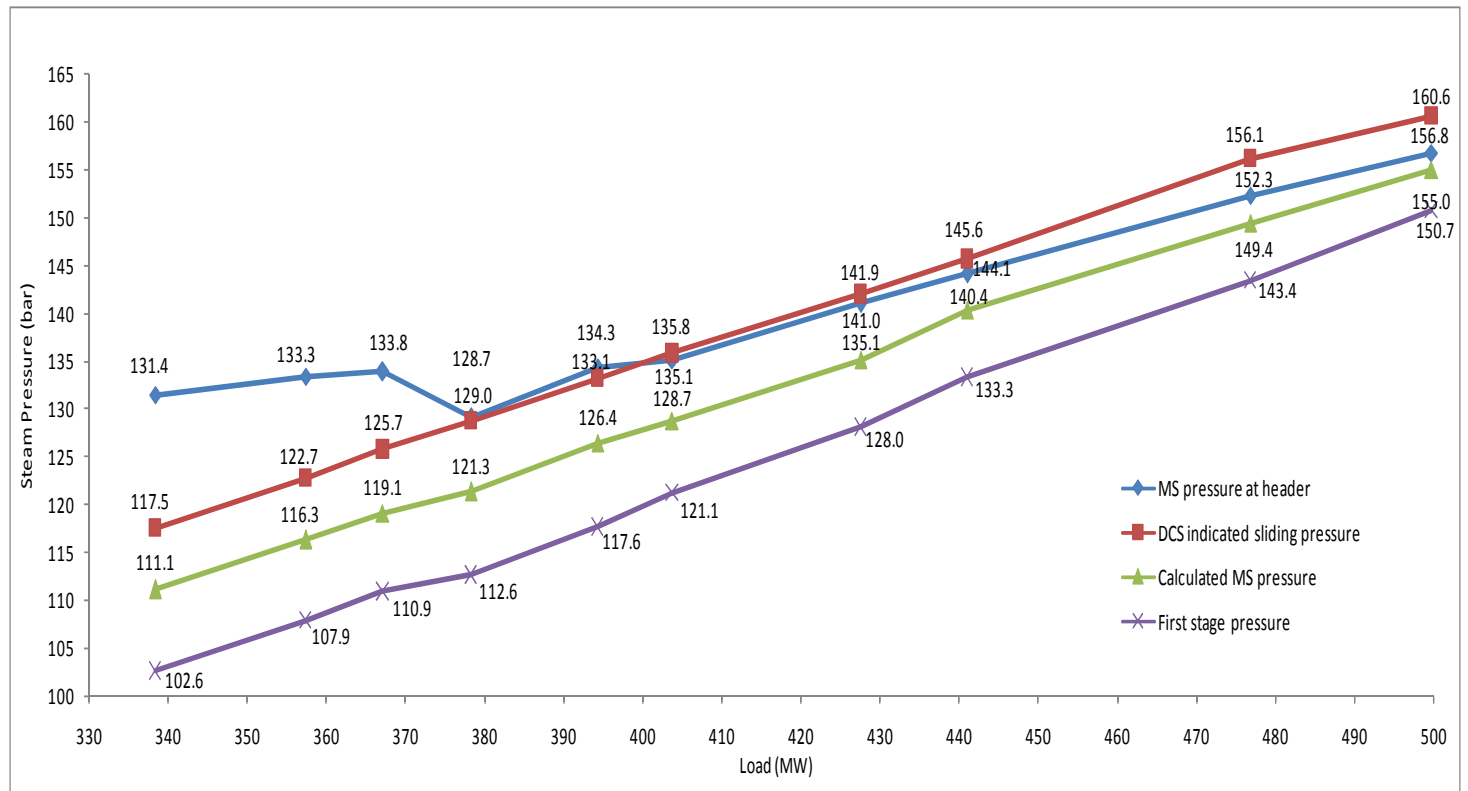

Fig.3. Variation between main steam pressure (maintained), DCS indicated sliding pressure, calculated main steam pressure and first stage pressure.

\section{ACKNOWLEDGMENT}

The author is grateful to the management and staff of HNPCL and SOMC for their cooperation and helpful discussions.

\section{REFERENCES}

[1] George Darie, Horia Peteu, Gabriel Negreanu, Viorel Gherghina, "Sliding pressure operation of large conventional steam power units", IASME, 2007. 
[2] P.K.Nag, Engineering Thermodynamics, Mc Graw Hill (ISBN-9780-07026-0627), fourth edition.

[3] Gerald Weber Commonwealth Edison company, "Sliding pressure analysis".

[4] Yong Hu, Ji-zhen Liu, De-Liang Zeng, Wei Wang, Ya-zhe Li, "The optimal steam pressure of thermal power plant in a given load", Energy and Power Engineering, 2013, 5, 278-282.

[5] Gerard Kosman, Henryk Lukowicz, krzysztof Nawrat, Wojciech Kosman, "Assessment of the effects of the operation of power units on sliding pressure", Eksploatacja Niezawodnosc NR 3/2007.

[6] S.C.Kaushik, V.Siva Reddy, S.K.Tyagi, "Energy and exergy anlaysis of thermal power plants: A review”, Renewable and Sustainable energy reviews 15(2011, ELSEVIER).

[7] Sairam Adibhatla, S.C.Kaushik, "Energy and exergy analysis of a super critical thermal power plant at various load conditions under constant and pure sliding pressure operation", Applied Thermal Engineering 73(2014), ELSEVIER.

[8] Ankur Geete, A.I. Khandwawala, "Thermodynamci analysis of $120 \mathrm{MW}$ thermal power plants with combined effect of constant inlet pressures (124.61 bar) and different inlet temperatures", Case studies in thermal engineering 1(2013), ELSEVIER.

[9] Marc A. Rosen, "Claryfying thermodynamics efficiencies and losses via exergy", Exergy, an International Journal 2(2002)3-5, ELSEVIER.

[10] Janulis, V.J, “ The effects of partial control valve opening on turbine heat rate", Commonwealth Edison efficiency division, Instr. No. 73.1956.

[11] Silvestri, G.J. JR-Aanstad, O.J.Ballantyne, J.J., “A review of sliding pressure for fossil fueled steam turbine generators”, Westinghouse Electric Corp., Lester, Penn., 1972.

[12] Robert A.Leffler, Craig R.Bradshaw, Eckhard A.Groll, Suresh V.Garimella, "Alternative heat rejection methods for power plants", Applied Energy 92(2012), ELSEVIER.

[13] Wei Wang, Lu Li, Dongteng Long, Tizhen Liu, Deliang Zenf, Can Cui, "Improved boiler-turbine coordinated control of $1000 \mathrm{MW}$ power units by introducing condensate throtting", Journal of Process control-50(2017), ELSEVIER.

[14] Wen Tan, Fang Fang, Liang Tian, Caifen Fu, Jizhen Liu, "Linear control of a boiler-turbine unit: Analysis and Design", ISA transactions 47(2008), ELSEVIER.

[15] Omendra Kumar Singh, S.C.Kaushik, "Variables influencing the exergy based performance of a steam power plant", International Journal of Green Energy 10:257-284, 2013, Taylor and Francis Group. 